\title{
Fusion dynamics around the Coulomb barrier
}

\author{
K. Hagino* ${ }^{*}$ N. Rowley ${ }^{\dagger}$, T. Ohtsuki**, M. Dasgupta ${ }^{\ddagger}$, J.O. Newton ${ }^{\ddagger}$ and \\ D.J. Hinde \\ *Yukawa Institute for Theoretical Physics, Kyoto University, Kyoto 606-8502, Japan \\ ${ }_{\dagger}^{\dagger}$ Institut de Recherches Subatomiques, UMR7500, IN2P3-CNRS/Universtité Louis Pasteur, BP28, \\ F-67037 Strasbourg Cedex 2, France \\ ${ }^{* *}$ Laboratory of Nuclear Science, Tohoku University, Sendai 982-0826, Japan \\ * Department of Nuclear Physics, Research School of Physical Sciences and Engineering, \\ Australian National University, Canberra ACT 0200, Australia
}

\begin{abstract}
.
We perform exact coupled-channels calculations, taking into account properly the effects of Coulomb coupling and the finite excitation energy of collective excitations in the colliding nuclei, for three Fm formation reactions, ${ }^{37} \mathrm{Cl}+{ }^{209} \mathrm{Bi},{ }^{45} \mathrm{Sc}+{ }^{197} \mathrm{Au}$, and ${ }^{59} \mathrm{Co}+{ }^{181} \mathrm{Ta}$. For the ${ }^{37} \mathrm{Cl}+$ ${ }^{209} \mathrm{Bi}$ and ${ }^{45} \mathrm{Sc}+{ }^{197} \mathrm{Au}$ reactions, those calculations well reproduce the experimental total fission cross sections, and a part of the extra-push phenomena can be explained in terms of the Coulomb excitations to multi-phonon states. On the other hand, for the heaviest system, the deep-inelastic collisions become much more significant, and the fission cross sections are strongly overestimated. We also discuss the surprisingly large surface diffuseness parameters required to fit recent highprecision fusion data for medium-heavy systems, in connection with the fusion supression observed in massive systems.
\end{abstract}

\section{INTRODUCTION}

The coupled-channels method has been very successful in reproducing experimental cross sections for heavy-ion reactions involving medium-heavy nuclei. Particularly, in many systems, it simultaneously reproduces the subbarrier enhancement of fusion cross sections and the shape of the fusion barrier distribution by including a few low-lying collective excitations of colliding nuclei and nucleon transfer channels [1]. It is now a standard theoretical tool to analyse experimental fusion and quasi-elastic cross sections at energies around the Coulomb barrier [2].

However, it has not yet been completely understood to what extent this method works for the fusion of massive systems, where the charge product of the target and projectile nuclei, $Z_{P} Z_{T}$, is typically larger than about 1800 . For those systems, other reaction processes, such as deep-inelastic collision and quasi-fission, come into play, and the reaction dynamics around the Coulomb barrier becomes much more complex than for medium-heavy systems [3]. In order to calculate fusion cross sections, the competition of fusion with these other processes has to be taken into account properly [4, 5, 6], and the dynamics after the Coulomb barrier is overcome becomes very important. This is the most difficult problem in the fusion of massive nuclei, and there are still large ambiguities in theoretical predictions of fusion cross sections. Because of this reason, one often employs a simplified coupled-channels treatment for the barrier penetration 
prior to the touching configuration [5, 7], which is essentially based on the constant coupling approximation [8] or a variant [9].

In this contribution, we critically examine the consequences of using such a simplified coupled-channels framework, and point out that the exact treatment for the Coulomb coupling plays an important role in massive systems. We then apply the exact coupledchannels approach to total fission cross sections for three Fm formation reactions [10], where the charge product $Z_{P} Z_{T}$ is given by $1411\left({ }^{37} \mathrm{Cl}+{ }^{209} \mathrm{Bi}\right), 1659\left({ }^{45} \mathrm{Sc}+{ }^{197} \mathrm{Au}\right)$, and $1971\left({ }^{59} \mathrm{Co}+{ }^{181} \mathrm{Ta}\right)$. We will show that the small hindrance of the reduced cross sections for the second heaviest system compared with the lightest system can be understood in terms of the effect of energy loss due to the Coulomb excitation, while the fusion hindrance for the heaviest system exceeds that effect and an explicit treatment of deepinelastic collisions is necessary. We also discuss the surface property of the nucleusnucleus potential, where recent high-precision fusion data for medium-heavy systems systematically show that a surprisingly large diffuseness parameter is required in order to fit them [11, 12, 13, 14]. We argue that this apparant anomaly could originate from the competition between fusion and the deep-inelastic processes which occur in fusion of heavy systems [14].

\section{COUPLED-CHANNELS APPROACH TO FUSION OF MASSIVE SYSTEMS}

\section{Effect of Coulomb excitations}

A hot compound nucleus formed by a fusion reaction decays either by emitting a few particles (mainly neutrons) and gamma rays, or by fission. The fusion cross section is thus a sum of the evaporation residue and the fusion-fission cross sections. Theoretically, it is computed as

$$
\sigma_{\text {fus }}(E)=\frac{\pi}{k^{2}} \sum_{l}(2 l+1) P_{\text {fus }}(E, l)=\frac{\pi}{k^{2}} \sum_{l}(2 l+1) T_{\mathrm{bp}}(E, l) \cdot P_{\mathrm{CN}}(E, l),
$$

where $T_{\mathrm{bp}}(E, l)$ is the barrier passing probability for the Coulomb barrier while $P_{\mathrm{CN}}(E, l)$ is the probability of compound nucleus formation after barrier penetration.

For medium-heavy systems, the compound nucleus is formed immediately after the Coulomb barrier is overcome, and $P_{\mathrm{CN}}$ is essentially 1 . This justifies the assumption of strong absorption inside the Coulomb barrier, or equivalently, the incoming wave boundary condition [2]. The coupled-channels approach has been successful here. For massive systems, on the other hand, $P_{\mathrm{CN}}$ significantly deviates from 1 , due to competition among several reaction processes. The fusion cross sections, therefore, appear to be hindered if one compares fusion cross sections with those obtained by assuming $P_{\mathrm{CN}}=1$. Recent experimental data clearly indicate the strong competition between compound nucleus formation and quasi-fission [15, 16, 17].

In Eq. (1), the barrier passing probability $T_{\mathrm{bp}}$ can in principle be computed by the coupled-channels approach. For this, although exact coupled-channels codes are available [2], a simplified approach has often been taken, even for fusion of massive systems 


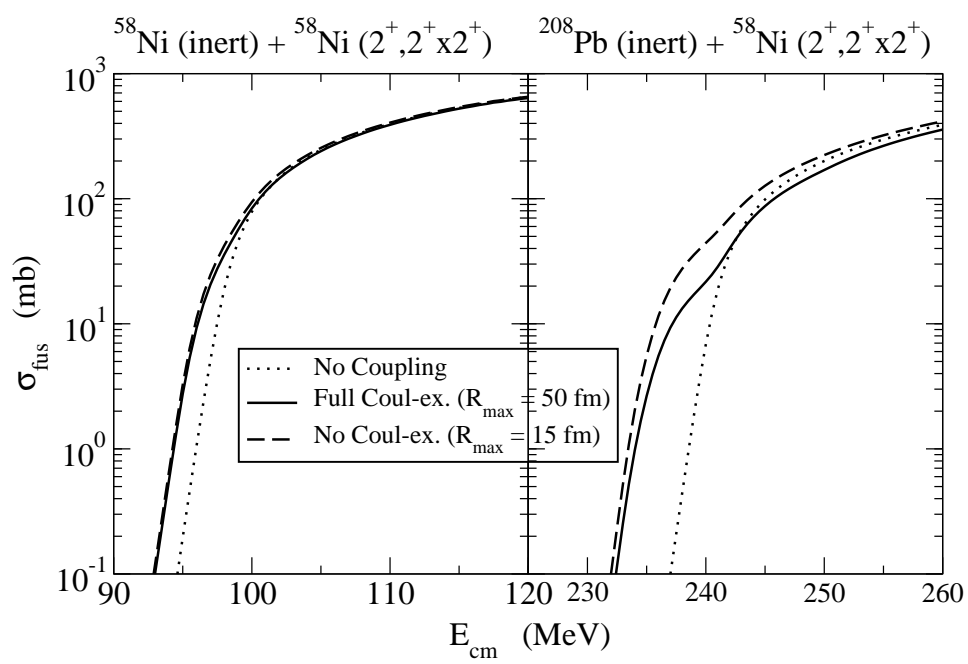

FIGURE 1. Influence of the Coulomb couplings in fusion cross sections for the ${ }^{58} \mathrm{Ni}+{ }^{58} \mathrm{Ni}$ (left panel) and the ${ }^{58} \mathrm{Ni}+{ }^{208} \mathrm{~Pb}$ (right panel) systems. The double quadrupole phonon excitation in the projectile nucleus ${ }^{58} \mathrm{Ni}$ is included in the coupled-channels calculations, while the target nucleus is treated as inert. The solid line includes the full effect of the Coulomb excitation, while the dashed line disregards it by matching the numerical wave functions to the asymptotic wave functions at relatively small distance. The fusion cross sections without the coupling are denoted by the dotted line.

[5, 7]. The simplification is achieved by using one or more of the following approximations: i) the linear coupling approximation, where the nuclear coupling potential is assumed to be linear with respect to an excitation operator for intrinsic motions, ii) multi-phonon excitations are neglected, and iii) the eigenchannel approximation, where the intrinsic excitation energies are treated approximately. Since the effective coupling strength is approximately proportional to $Z_{P} Z_{T}$ for a given value of deformation parameter [18], the shortcomings of these approximations become more severe for heavier systems.

The validity of the first and the second approximations has been examined in detail in Refs. [18, 19, 20]. We therefore discuss the third point here. The eigenchannel approach is intimately related to the concept of barrier distribution [1, 8, 21]. Although this approach is exact when the intrinsic excitation energy vanishes, it also works well even with a finite excitation energy as long as the coupling potential is localized inside the uncoupled barrier [22]. In realistic cases, however, the coupling potential extends outside the barrier due to the long range Coulomb interaction. If, prior to reaching the barrier, there is appreciable Coulomb excitation to a collective state whose excitation energy is not small, this results in a decrease of the relative energy, leading to the reduction of fusion cross sections and a modification of effective eigen-barriers. Since the eigenchannel approach treats the excitation energy approximately, this effect will be missed in a calculation. 


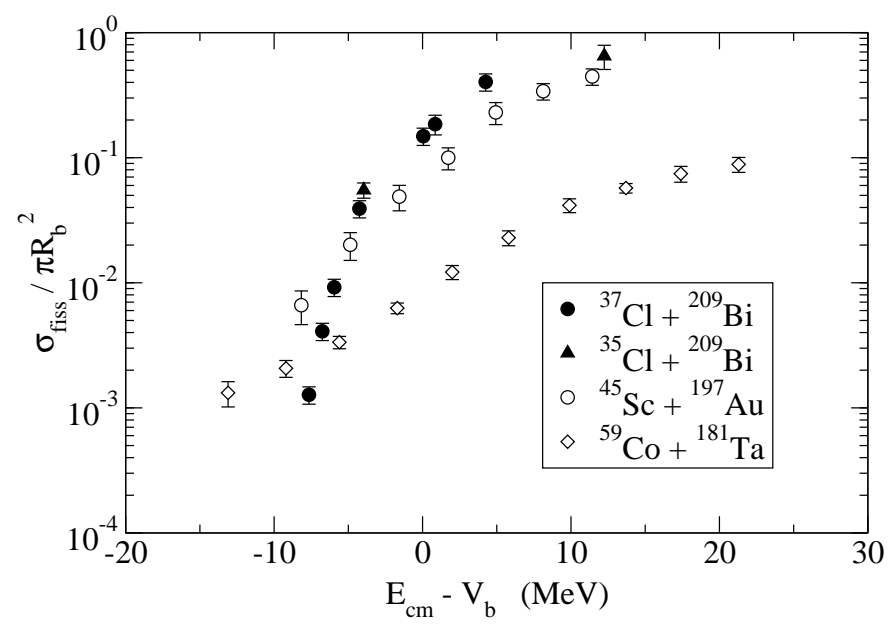

FIGURE 2. Reduced total fission cross sections for four reactions forming $\mathrm{Fm},{ }^{35,37} \mathrm{Cl}+{ }^{209} \mathrm{Bi}$ (the filled circles and triangles), ${ }^{45} \mathrm{Sc}+{ }^{197} \mathrm{Au}$ (the open circles), and ${ }^{59} \mathrm{Co}+{ }^{181} \mathrm{Ta}$ (the open diamonds), as a function of energy measured from the Coulomb barrier height.

In order to demonstrate the effect of Coulomb excitations prior to the barrier, figure 1 compares fusion cross sections for the ${ }^{58} \mathrm{Ni}+{ }^{58} \mathrm{Ni}$ system with those for the ${ }^{58} \mathrm{Ni}+$ ${ }^{208} \mathrm{~Pb}$ system. We assume that the target nucleus is inert, and include only the double quadrupole phonon excitation in the projectile ${ }^{58} \mathrm{Ni}$ nucleus. The excitation energy for the single phonon state is $1.45 \mathrm{MeV}$, and we assume a simple harmonic oscillator coupling for the double phonon excitation. The solid and dashed lines are obtained by integrating the coupled-channels equations from inside the Coulomb barrier to $R_{\max }=50$ $\mathrm{fm}$ and $15 \mathrm{fm}$, respectively. The latter calculation, therefore, effectively disregards the effect of Coulomb excitation outside the Coulomb barrier. For a comparison, we also show the fusion cross sections in the no coupling limit by the dotted line. One clearly sees that the Coulomb coupling considerably alters the fusion cross sections when the target is heavy, while the difference is small for the lighter system. One also notices that the fusion cross sections are even smaller than the no coupling calculations for the heavier system at energies above the Coulomb barrier. Evidently, the Coulomb excitation provides another mechanism of fusion inhibition in massive systems.

\section{Total fission cross sections for $\mathrm{Fm}$ formation reactions}

Let us now discuss fission cross sections for $Z=100(\mathrm{Fm})$ formation reactions measured by Ohtsuki et al. [10]. The experimental cross sections were obtained by selecting the fission events in the TOF vs $\Delta E$ plot, and thus contain both the fusion-fission and the quasi-fission (if any) cross sections. For the heaviest system, the fission events were 


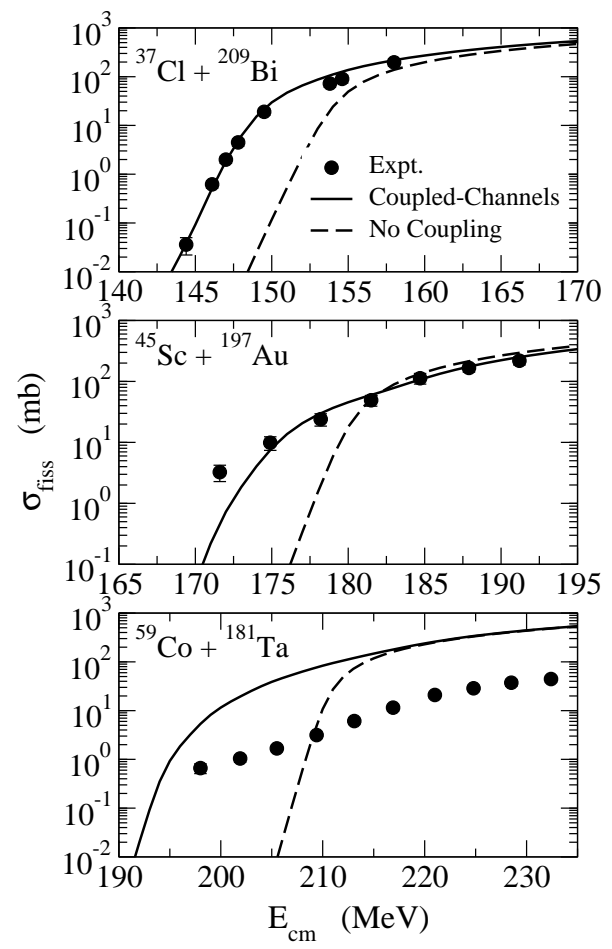

FIGURE 3. Comparison of the coupled-channels calculations with the experimental data for total fission cross sections for the Fm formation reactions. The solid line is the result of the coupled-channels calculations, while the dashed line is obtained without any coupling. The experimental data are denoted by the filled circles.

not well separated from the deep-inelastic collision (DIC) events. For this system, the fission cross sections were estimated by choosing the same mass region of fission fragments as that observed in the ${ }^{35,37} \mathrm{Cl}+{ }^{209} \mathrm{Bi}$ reaction. Therefore, the experimental fission cross sections might be underestimated for the heaviest system if the fission events extend towards the DIC region. Fig. 2 shows the observed reduced fission cross sections as a function of the difference between the center of mass energy and the average barrier energy for four systems, ${ }^{35,37} \mathrm{Cl}+{ }^{209} \mathrm{Bi}\left(Z_{P} Z_{T}=1411\right),{ }^{45} \mathrm{Sc}+{ }^{197} \mathrm{Au}\left(Z_{P} Z_{T}=1659\right)$, and ${ }^{59} \mathrm{Co}+{ }^{181} \mathrm{Ta}\left(Z_{P} Z_{T}=1971\right)$. Notice that the reduced cross sections for the second heaviest system $\left({ }^{45} \mathrm{Sc}+{ }^{197} \mathrm{Au}\right)$ are somewhat smaller than those for the lightest system $\left({ }^{35,37} \mathrm{Cl}+{ }^{209} \mathrm{Bi}\right)$, and the cross sections for the heaviest system $\left({ }^{59} \mathrm{Co}+{ }^{181} \mathrm{Ta}\right)$ are substantially hindered compared with the other two systems.

In order to investigate whether these features can be understood in terms of the Coulomb excitation discussed in the previous subsection, we perform exact coupledchannels calculations. For this purpose, we use an extended version of the computer code CCFULL [2], where the effect of finite ground state spin of colliding nuclei is incorporated within the isocentrifugal approximation. For the ${ }^{37} \mathrm{Cl}+{ }^{209} \mathrm{Bi}$ system, we include three vibrational states, $5 / 2^{+}(3.09 \mathrm{MeV}), 7 / 2^{-}(3.1 \mathrm{MeV})$, and $9 / 2^{-}(4.01 \mathrm{MeV})$ 
in ${ }^{37} \mathrm{Cl}$ as well as double octupole vibrations in ${ }^{209} \mathrm{Bi}$. The deformation parameters are estimated from the experimental $\mathrm{B}(\mathrm{E} 2)$ and $\mathrm{B}(\mathrm{E} 3)$ values. We introduce a single effective channel for the seven octupole states which have a character of $h_{9 / 2} \otimes{ }^{208} \mathrm{~Pb}\left(3^{-}\right)$in ${ }^{209} \mathrm{Bi}$, and consider a harmonic oscillator coupling for the double phonon state. For the ${ }^{45} \mathrm{Sc}+$ ${ }^{197} \mathrm{Au}$ system, we include 5 quadrupole states which have a character of $f_{7 / 2} \otimes{ }^{44} \mathrm{Ca}\left(2^{+}\right)$ in ${ }^{45} \mathrm{Sc}\left[3 / 2^{-}(0.38 \mathrm{MeV}), 5 / 2^{-}(0.72 \mathrm{MeV}), 11 / 2^{-}(1.24 \mathrm{MeV}), 7 / 2^{-}(1.41 \mathrm{MeV})\right.$, and $\left.9 / 2^{-}(1.66 \mathrm{MeV})\right]$, and treat the target nucleus ${ }^{197} \mathrm{Au}$ as a classical rotor with $\beta_{2}=-0.13$ and $\beta_{4}=-0.03$. For ${ }^{45} \mathrm{Sc}$, since the excitation energies for the quadrupole states are not close to each other, we do not introduce an effective single channel, but treat them exactly. For the ${ }^{59} \mathrm{Co}+{ }^{181} \mathrm{Ta}$ system, we include 5 quadrupole states which have a character of $\left(f_{7 / 2}\right)^{-1} \otimes{ }^{60} \mathrm{Ni}\left(2^{+}\right)$in ${ }^{59} \mathrm{Co}\left[3 / 2^{-}(1.1 \mathrm{MeV}), 9 / 2^{-}(1.19 \mathrm{MeV}), 11 / 2^{-}\right.$ (1.46 MeV), $5 / 2^{-}(1.48 \mathrm{MeV})$, and $\left.7 / 2^{-}(1.74 \mathrm{MeV})\right]$, and treat the target nucleus ${ }^{181} \mathrm{Ta}$ as a classical rotor with $\beta_{2}=0.354$ and $\beta_{4}=-0.05$. More details of the calculations will be given elsewhere [23].

The results of those calculations are shown in Fig. 3. One finds that the coupledchannels calculations well reproduce the experimental data for the two lightest systems, ${ }^{37} \mathrm{Cl}+{ }^{209} \mathrm{Bi}$ and ${ }^{45} \mathrm{Sc}+{ }^{197} \mathrm{Au}$. Especially, the reduction of cross sections in the latter system is reproduced nicely. As we discussed in the previous subsection, this small hindrance of cross sections is caused by the strong Coulomb coupling to the collective states outside the Coulomb barrier. In contrast, the coupled-channels calculation considerably overestimates fission cross sections for the heaviest system, ${ }^{59} \mathrm{Co}+{ }^{181} \mathrm{Ta}$. We will discuss this point in the next subsection.

\section{Role of deep-inelastic collision}

In his review article, Reisdorf argued [3] that cross sections for the sum of fusion and other damped reactions may be interpreted as the total barrier passing cross sections. Indeed, he found that the sum of fusion and deep-inelastic collision (DIC) cross sections for the ${ }^{58} \mathrm{Ni}+{ }^{124} \mathrm{Sn}$ reaction could be well reproduced by the standard potential model (see Fig. 4). More recently, Esbensen et al. followed a similar idea and reproduced the experimental cross sections for the sum of fusion and DIC reactions for the same system with the coupled-channels approach [25]. In the semiclassical picture, deep inelastic collisions correspond to those trajectories which overcome the barrier in the entrance channel but eventually escape after appreciable interaction with the target. All of those considerations immediately lead to the idea that the total barrier passing cross sections, which the coupled-channels approach yields, may have to be compared with a sum of fusion, quasi-fission, and DIC, i.e.,

$$
\sigma_{\mathrm{bp}}(E)=\sigma_{\text {fus }}(E)+\sigma_{\mathrm{qf}}(E)+\sigma_{\mathrm{DIC}}(E) \text {. }
$$

The large reduction of total fission cross sections for the ${ }^{59} \mathrm{Co}+{ }^{181} \mathrm{Ta}$ system, therefore, may be attributed to the competition between fusion, quasi-fission and DIC reactions.

The present framework of the coupled-channels method could be used to obtain inclusive cross sections of fusion and DIC reactions, but it would be difficult to obtain them 


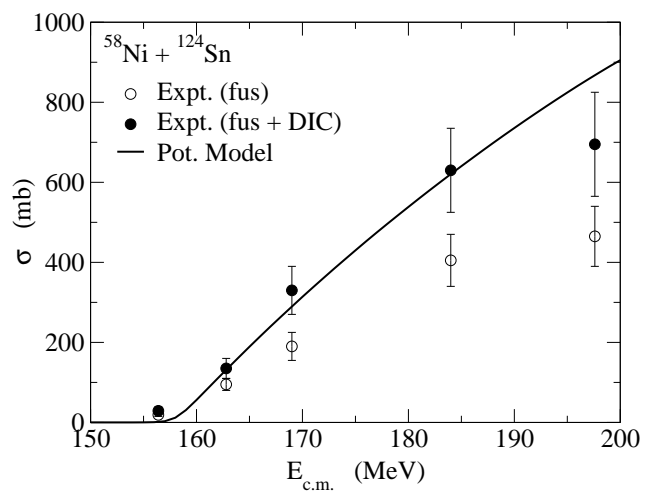

FIGURE 4. Fusion cross sections (the open circles) and the sum of fusion and deep inelastic collision cross sections (the filled circles) for the ${ }^{58} \mathrm{Ni}+{ }^{124} \mathrm{Sn}$ reaction. The solid line is a prediction of the barrier penetration model with a global nucleus-nucleus potential. The experimental data are taken from Ref. [24].

separately. One possible way to isolate fusion from DIC theoretically is to introduce angular momentum truncation in Eq. (1) and exclude higher partial wave contributions, as was done by Zagrebaev et al. [5]. However, a large ambiguity exists in this prescription, since there is no clear and unique way to introduce the angular momentum truncation, especially at energies below the barrier [26]. In recent publications, Abe et al. combined the surface friction model with the Langevin approach in order to take into account the competition between fusion and DIC in the approaching phase for a formation reaction of superheavy elements [4]. This approach may be promising, but is essentially classical. Computation of fusion cross sections with a quantum mechanical model under the influence of DIC process is still an open problem.

\section{SURFACE DIFFUSENESS ANOMALY IN FUSION POTENTIALS}

Let us now discuss the second subject, that is the surface property of the nucleus-nucleus potential and its anomaly, recently recognised in fusion reactions for medium-heavy systems. For scattering processes, it seems well accepted that the surface diffuseness parameter $a$ should be around $0.63 \mathrm{fm} \mathrm{[27]} \mathrm{if} \mathrm{the} \mathrm{nuclear} \mathrm{potential} \mathrm{is} \mathrm{parametrised} \mathrm{by} \mathrm{a}$ Woods-Saxon (WS) form. In marked contrast, recent high-precision fusion data suggest that a much larger diffuseness, between 0.8 and $1.4 \mathrm{fm}$, is needed to fit the data [11, 12]. This is not just for particular systems but seems to be a rather general result [14].

We illustrate this problem in Fig. 5 by comparing experimental data for the ${ }^{16} \mathrm{O}+$ ${ }^{208} \mathrm{~Pb}$ fusion reaction with various coupled-channels calculations with the WS potential. We include the double octupole phonon and the single $5^{-}$phonon excitations in ${ }^{208} \mathrm{~Pb}$. At energies well above the barrier, where the fusion cross sections $\sigma_{\text {fus }}$ is relatively in- 


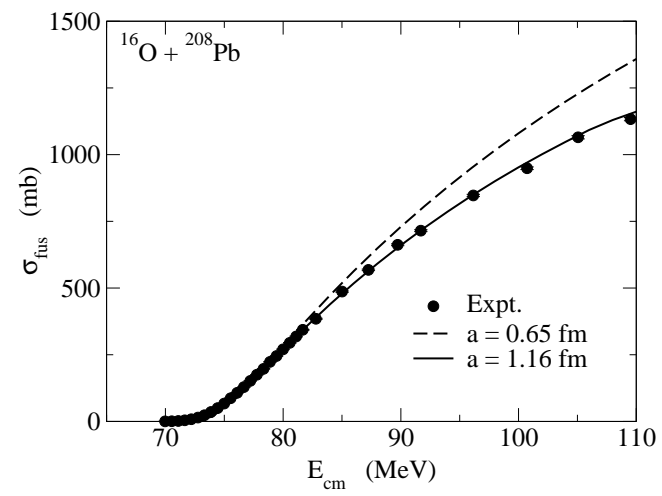

FIGURE 5. Comparison of coupled-channels calculations with the experimental data for the ${ }^{16} \mathrm{O}+$ ${ }^{208} \mathrm{~Pb}$ fusion reaction. The double octupole phonon as well as the single $5^{-}$phonon excitations are included. The solid and the dashed lines are obtained by setting the surface diffuseness parameter of Woods-Saxon potential to be $a=1.16$ and $a=0.65 \mathrm{fm}$, respectively. The experimental data are taken from Ref. [28].

sensitive to the couplings, a WS potential with a diffuseness $a=0.65 \mathrm{fm}$ significantly overestimates fusion cross sections (dashed line). Changing the depth and radius parameters in the WS potential is not helpful, since it merely leads to an energy shift in the calculated fusion cross sections without significantly changing the energy dependence. On the other hand, a potential with $a=1.16 \mathrm{fm}$ (full line) fits the data well. A similar problem also exists at energies below the barrier [12, 13], but we do not discuss it in this contribution.

Up to now, several possible reasons for this anomaly have been considered. These include the departure of the nuclear potential from the WS form [11, 12], dissipation effects [12], and unrecognised systematic errors in experimental data [14], but none of them is conclusive yet. We would like to propose here another possible effect, that is, the competition between fusion and deep inelastic collision [14]. This is motivated by an apparent similarity between fusion inhibition in massive systems discussed in the previous section (see fig. 4) and the overestimate of fusion cross sections shown in fig. 5 . To this end, let us introduce a suppression factor $S$ which is defined as a ratio between the experimental fusion cross sections and the prediction of the potential model with the standard value for the diffuseness parameter, $a \approx 0.63 \mathrm{fm}$. Provided that $S$ is independent of bombarding energy, it can be stated that the fusion cross sections are hindered by a factor $S$ for whatever reason.

The values of $S$ obtained for a large number of systems are shown in fig. 6 [14]. Also shown are two points (large filled stars) at $Z_{1} Z_{2}=1400$ derived from the data for the ${ }^{58} \mathrm{Ni}+{ }^{112,124} \mathrm{Sn}$ reactions at energies around the fusion barrier. For these systems, as we indicate in Fig. 4, a very significant contribution from deep-inelastic scattering has been observed experimentally even at energies below the barrier [24]. In this case, the value of $S$ has been taken as $\sigma_{\text {fus }}$ divided by the sum of $\sigma_{\text {fus }}$ and $\sigma_{\text {DIC. }}$. These two points do not 


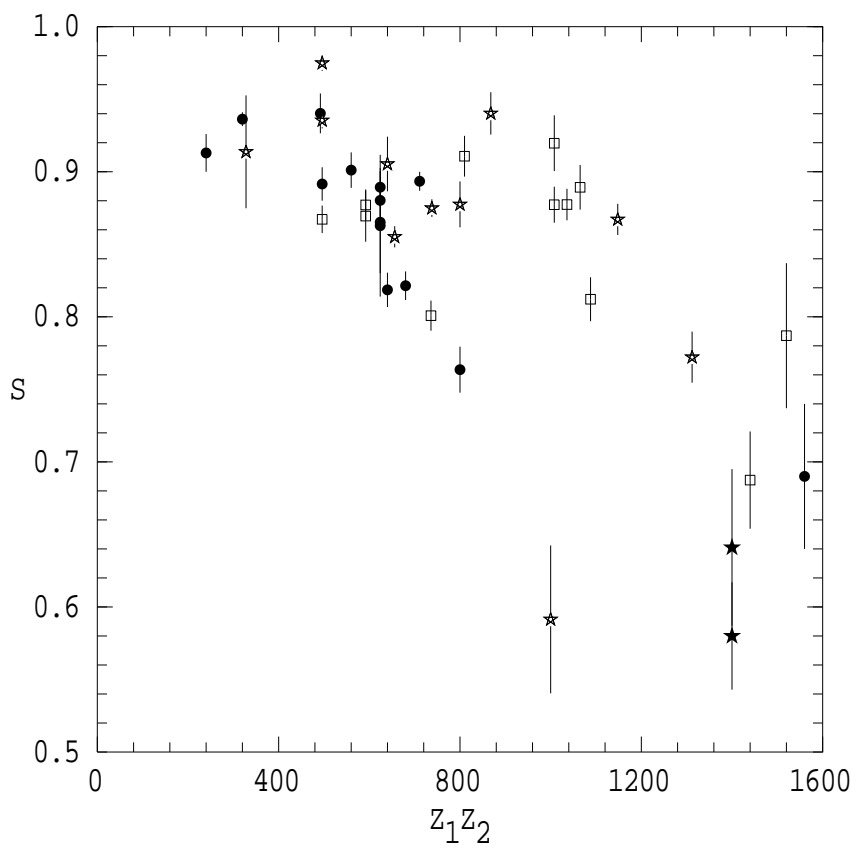

FIGURE 6. Calculated suppression factors $S$ for fusion cross sections with respect to the prediction of potential model with $a \approx 0.63 \mathrm{fm}$. The large filled stars at $Z_{1} Z_{2}=1400$ refer to the reactions ${ }^{58} \mathrm{Ni}+$ ${ }^{112,124} \mathrm{Sn}$, where the deep-inelastic collisions have been observed experimentally.

seem inconsistent with the other points with large $Z_{1} Z_{2}$ in Fig. 6, which are derived from the fusion data only. Strongly damped reactions similar to DIC are reported to occur in lighter systems, for example, ${ }^{32} \mathrm{~S}+{ }^{64} \mathrm{Ni}$ [29] $\left(Z_{1} Z_{2}=448\right)$ at energies well above the barrier. It would be interesting to see if they also occur at energies closer to the barrier. If they do, the values of $S$ would be reduced below unity and might result in at least a partial explanation of the experimental values for $a$ being much larger than the standard value, $0.63 \mathrm{fm}$.

\section{SUMMARY}

Extensive efforts have been made both experimentally and theoretically to understand fusion of massive systems, especially for synthesis of superheavy elements (SHE), but the reaction mechanism has not yet been completely understood. Here, we have performed coupled-channels calculations and pointed out that the exact treatment of Coulomb excitation becomes important for massive systems. We have applied the coupled-channels framework to three Fm formation reactions, and have shown that Coulomb excitation indeed provides an important mechanism of inhibition of fusion cross sections for transitional systems ("pre-SHE" systems) between medium-heavy and SHE regions. For SHE systems, we have argued that the deep-inelastic collision needs to be taken into account explicitly in theoretical models. However, it is still an open problem of how to incorporate the competition between fusion and DIC quantum 
mechanically, and further developments will be required. We have also discussed the large surface diffuseness problem observed in the recent high precision measurements of fusion cross sections for medium-heavy systems in connection with the fusion hindrance in massive systems. We have argued that the competition between fusion and deep-inelastic collision may provide a promising avenue to explain this anomaly. In this connection, it would be interesting to study, both experimentally and theoretically, light systems near to the fusion barrier to see whether fusion was inhibited by the presence of DIC. Especially, theoretical calculations involving friction for light systems would be of great interest, since experimental measurements are likely to be difficult when the fusion suppression factor appoaches unity.

\section{REFERENCES}

1. M. Dasgupta, D.J. Hinde, N. Rowley, and A.M. Stefanini, Annu. Rev. Nucl. Part. Sci. 48, 401 (1998).

2. K. Hagino, N. Rowley, and A.T. Kruppa, Comp. Phys. Comm. 123, 143 (1999).

3. W. Reisdorf, J. Phys. G20, 1297 (1994).

4. C. Shen, G. Kosenko, and Y. Abe, Phys. Rev. C66, 061602(R) (2002); B. Bourquet, Y. Abe, and G. Kosenko, e-print: nucl-th/0308019

5. V.I. Zagrebaev, Y. Aritomo, M.G. Itkis, Yu. Ts. Oganessian, and M. Ohta, Phys. Rev. C65, 014607 (2002).

6. Y. Aritomo, T. Wada, M. Ohta, and Y. Abe, Phys. Rev. C59, 796 (1999).

7. V.Yu. Denisov and S. Hofmann, Phys. Rev. C61, 034606 (2000).

8. C.H. Dasso, S. Landowne, and A. Winther, Nucl. Phys. A405, 381 (1983); A407, 221 (1983).

9. M. Dasgupta et al., Nucl. Phys. A539, 351 (1992).

10. Y. Nagame et al. , Japan Atomic Energy Res. Inst. Tandem VDG Ann. Rept., 1993, p. 45 (1994); T. Ohtsuki et al., ibid p. 46.

11. J.O. Newton, C.R. Morton, M. Dasgupta, J.R. Leigh, J.C. Mein, D.J. Hinde, H. Timmers, and K. Hagino, Phys. Rev. C64, 064608 (2001).

12. K. Hagino, M. Dasgupta, I.I. Gontchar, D.J. Hinde, C.R. Morton, and J.O. Newton, Proc. Fourth Italy-Japan Symposium on Heavy-Ion Physics, Tokyo, Japan (World Scientific, Singapore, 2002), p. 87; e-print nucl-th/0110065

13. K. Hagino, N. Rowley, and M. Dasgupta, Phys. Rev. C67, 054603 (2003).

14. J.O. Newton, R.D. Butt, M. Dasgupta, I.I. Gontchar, D.J. Hinde, C.R. Morton, A. Mukherjee, and K. Hagino (to be published).

15. A.C. Berriman, D.J. Hinde, M. Dasgupta, C.R. Morton, R.D. Butt, and J.O. Newton, Nature 413, 144 (2001).

16. D.J. Hinde, M. Dasgupta, and A. Mukherjee, Phys. Rev. Lett. 89, 282701 (2002).

17. R.N. Sagaidak et al., Phys. Rev. C68, 014603 (2003); A. Yu. Chizhov et al., Phys. Rev. C67, 011603 (2003).

18. K. Hagino, N. Takigawa, M. Dasgupta, D.J. Hinde, and J.R. Leigh, Phys. Rev. C55, 276 (1997).

19. K. Hagino, N. Takigawa, M. Dasgupta, D.J. Hinde, and J.R. Leigh, Phys. Rev. Lett. 79, 2014 (1997).

20. A.M. Stefanini et al., Phys. Rev. Lett. 74, 864 (1995).

21. N. Rowley, G.R. Satchler, and P.H. Stelson, Phys. Lett. B254, 25 (1991).

22. K. Hagino, N. Takigawa, and A.B. Balantekin, Phys. Rev. C56, 2104 (1997).

23. T. Ohtsuki, H. Ikezoe, Y. Nagame, J. Kasagi, and K. Hagino (to be published).

24. F.L.H. Wolfs, W. Henning, K.E. Rehm, and J.P. Schiffer, Phys. Lett. B196, 113 (1987); F.L.H. Wolfs, Phys. Rev. C36, 1379 (1987).

25. H. Esbensen, C.L. Jiang, and K.E. Rehm, Phys. Rev. C57, 2401 (1998).

26. C.H. Dasso and G. Pollarolo, Phys. Rev. C39, 2073 (1989).

27. R.A. Broglia and A. Winther, Heavy Ion Reactions (Addison-Wesley, Redwood City, CA, 1991).

28. C.R. Morton et al., Phys. Rev. C60, 044608 (1999).

29. G. Russo et al., Phys. Rev. C39, 2462 (1989). 